\title{
Avaliação dos critérios de diagnóstico das doenças pulpares e periapicais realizados pelos cirurgiões-dentistas da atenção básica do Município de Arcoverde/PE
}

\author{
Evaluation of the criteria for diagnosis of pulp and periapical diseases performed by dentists in
} primary care in the Municipality of Arcoverde/PE

Evaluación de los criterios de diagnóstico de las enfermedades pulpares y periapicales realizadas por los cirujanos dentistas de atención primaria en el Municipio de Arcoverde/PE

Bianca Maria Beserra Costa ORCID: https://orcid.org/0000-0002-8132-9881 Universidade de Pernambuco, Brasil E-mail: bianca.bcosta@upe.br

Mateus Queiroz Freire Leão ORCID: https://orcid.org/0000-0003-1424-7998

Universidade de Pernambuco, Brasil

E-mail: mateusleaodonto@gmail.com Felipe Bernardo Sena ORCID: https://orcid.org/0000-0002-6002-4704 Universidade de Pernambuco, Brasil E-mail: felipeodontoupe@gmail.com

Sandra Maria Alves Sayão Maia ORCID: https://orcid.org/0000-0003-3172-2332 Universidade de Pernambuco, Brasil E-mail: sandrinhasayao@hotmail.com

Paulo Maurício Reis de Melo Júnior ORCID: https://orcid.org/0000-0001-9926-5348 Universidade de Pernambuco, Brasil E-mail: Paulo.reis@upe.br

Isabel Maria de Araúo Pinto

ORCID: https://orcid.org/0000-0001-7037-7215

Faculdade de Odontologia do Recife, Brasil E-mail: isabelaraujop@ hotmail.com

Natália Gomes de Oliveira

ORCID: https://orcid.org/0000-0001-6937-1537 Universidade de Pernambuco, Brasil E-mail: nataliagomes04@hotmail.com

Luís Felipe Espíndola-Castro ORCID: https://orcid.org/0000-0002-1923-8057 Universidade de Pernambuco, Brasil E-mail: lipe_espindola@hotmail.com

Pâmella Recco Álvares ORCID: https://orcid.org/0000-0003-3396-9339 Universidade de Pernambuco, Brasil

E-mail: pamella.recco@upe.br

Jorge Miguel Albrecht Ribeiro Filho

ORCID: https://orcid.org/0000-0002-3772-1851

Faculdade de Odontologia do Recife, Brasil

E-mail: Jorge_albrechtfilho@hotmail.com

\begin{abstract}
Resumo
O diagnóstico correto é o ponto chave no tratamento de qualquer enfermidade evitando imprevistos, complicações e agravos. Na endodontia, o diagnóstico das patologias pulpares e periapicais são realizados na atenção básica odontológica do serviço público brasileiro de saúde. Visando a melhoria do serviço de saúde bucal, este estudo teve como objetivo avaliar a conduta dos cirurgiões-dentistas do município de Arcoverde \PE no diagnóstico das alterações pulpares e periapicais. Foi realizado um estudo quantitativo-descritivo, por meio de questionário online aplicado aos profissionais das Unidades Básicas de Saúde. Após organização e categorização, os resultados obtidos revelaram que a maior parte dos profissionais são pós-graduados $(88,9 \%)$, concursados $(77,8 \%)$ e possuem menos de 3 anos de atuação na atenção básica do município de Arcoverde (66,66\%). Existe alta frequência do diagnóstico das alterações pulpares e periapicais nas UBSFs $(39,74 \%)$. Os profissionais se considerarão aptos para o reconhecimento da maioria
\end{abstract}


dos aspectos clínicos das doenças pulpares e periapicais $(85,47 \%)$ e para o diagnóstico endodôntico $(61,11 \%)$. Apenas $36,56 \%$ foi a média de acertos para a identificação das características clínicas, 35,07 \% dos aspectos radiográficos e $35,69 \%$ das respostas aos testes semiotécnicos. De acordo com a metodologia empregada, pode-se concluir que a maioria dos cirurgiões-dentistas da atenção básica do município de Arcoverde/PE possuem conhecimento insuficiente e utilizam de forma deficiente os critérios de diagnóstico das doenças pulpares e periapicais.

Palavras-chave: Diagnóstico; Doenças da polpa dentária; Doenças periapicais; Endodontia.

\begin{abstract}
Correct diagnosis is the key point in the treatment of any disease, avoiding unforeseen events, complications, and injuries. In Endodontics, the diagnosis of pulp and periapical pathologies is carried out with basic dental care provided by the Brazilian public health service. Aiming at improving the oral health service, this study aimed to evaluate the conduct of dental surgeons in the municipality of ArcoverdePE in the diagnosis of pulpal and periapical changes. A quantitative-descriptive study was carried out by means of an online questionnaire applied to the professionals of the Unidades Básicas de Saúde (Basic Health Units). After organization and categorization, the results revealed that most professionals have a postgraduate degree (88.9\%), are permanent employees $(77.8 \%)$, and have less than 3 years of experience in primary care in the municipality of Arcoverde (66.66\%). There is a high frequency of diagnosis of pulp and periapical alterations in UBSFs (39.74\%). Professionals considered themselves capable of recognizing more clinical aspects of pulpal and periapical diseases (85.47\%) and endodontic diagnosis (61.11\%). Only $36.56 \%$ were the mean value of correct answers for the identification of clinical features, 35.07\% for radiographic aspects and $35.69 \%$ of the answers to the semiotechnical tests. According to the methodology employed, it can be concluded that most dentists in primary care in the municipality of Arcoverde/PE have insufficient knowledge and poorly use the criteria for diagnosis of pulp and periapical diseases.
\end{abstract}

Keywords: Diagnosis; Dental pulp deseases; Periapical diseases; Endodontics.

\title{
Resumen
}

El diagnóstico correcto es el punto clave en el tratamiento de cualquier enfermedad, evitando imprevistos, complicaciones y lesiones. En endodoncia, el diagnóstico de patologías pulpares y periapicales se realiza en la atención odontológica proporcionada por el servicio público de salud brasileño. Con el objetivo de mejorar el servicio de salud bucal, este estudio tuvo como objetivo evaluar la conducta de los cirujanos dentistas del municipio de ArcoverdelPE en el diagnóstico de alteraciones pulpares y periapicales. Se realizó un estudio cuantitativo-descriptivo, mediante un cuestionario online aplicado a los profesionales de las Unidades Básicas de Salud. Tras la organización y categorización, los resultados obtenidos revelaron que la mayoría de los profesionales son postgraduados (88,9\%), licitados $(77,8 \%)$ y tienen menos de 3 años de experiencia en atención primaria en el municipio de Arcoverde $(66,66 \%)$. Existe una alta frecuencia de diagnóstico de cambios pulpares y periapicales en las UBSF (39,74\%). Los profesionales se considerarán capaces de reconocer la mayoría de los aspectos clínicos de las enfermedades pulpares y periapicales $(85,47 \%)$ y para el diagnóstico endodóntico $(61,11 \%)$. Sólo el 36,56\% fue el promedio de aciertos para la identificación de características clínicas, el 35,07\% de aspectos radiográficos y el 35,69\% de respuestas a pruebas semiotécnicas. De acuerdo con la metodología utilizada, se puede concluir que la mayoría de los odontólogos de atención primaria del municipio de Arcoverde/PE tienen un conocimientos insuficientes y utilizan los criterios de diagnósticos de enfermedades pulpares y periapicales de manera deficiente.

Palabras clave: Diagnóstico; Enfermedades de la pulpa dental; Enfermedades periapicales; Endodoncia.

\section{Introdução}

O campo da Odontologia que se dedica na prevenção, diagnóstico e tratamento das doenças que afetam a polpa dentária, a cavidade pulpar e os tecidos periodontais próximos, é a Endodontia (Campos, Campos, \& Bellei, 2018). No tratamento endodôntico é essencial que o sistema de canais radiculares esteja limpo, sendo isto alcançado com a erradicação dos microrganismos que prejudicam à cicatrização dos tecidos, possibilitando ao dente a recuperação e restabelecimento da sua função (Marques, 2014).

O componente pulpar de um dente hígido e íntegro é isolado das substâncias exógenas da cavidade bucal. Entretanto, cáries, infecções dos tecidos periodontais, alterações degenerativas, traumas dentários e procedimentos restauradores invadem a integridade dos tecidos que protegem a polpa, permitindo assim que as alterações no complexo dentino-pulpar ocorram, conduzindo, possivelmente, a uma doença pulpar e periapical. Sendo assim, o diagnóstico dessas doenças se torna essencial para um tratamento de excelência e a definição do melhor tratamento possível para cada caso (Albuquerque et al., 2011). 
Um plano de tratamento endodôntico bem-sucedido depende de um diagnóstico correto. Nas alterações da polpa dentária humana, os informes necessários para o estabelecimento das suas condições patológicas ficam restritos à anamnese, exame clínico, testes de sensibilidade pulpar e avaliação radiográfica. Isto ocorre pelo fato da polpa se encontrar envolvida por paredes de dentina, fato que impede sua visualização direta pelo profissional durante o atendimento clínico (Silva, Albergaria, Gonçalves, \& Santos, 2008).

Desse modo, dominar a arte do diagnóstico significa aprender os princípios da coleta e interpretação de dados, registrando minuciosamente os sinais e sintomas por meio da análise dos mesmos, e estabelecer um correto diagnóstico levando a uma condução adequada do tratamento (Medeiros et al., 2017).

Para tanto, é preciso que o cirurgião-dentista esteja preparado e detenha os conhecimentos necessários para discernir sobre a enfermidade e tratá-la. (Caputo, Bazzo, Silva, \& Daruge Júnior, 2010). Pois, uma alteração pulpar não tratada pode evoluir para a necrose pulpar. A necrose pulpar consiste na completa cessação dos processos metabólicos do tecido pulpar, e se não for removido do interior do canal radicular, os produtos tóxicos bacterianos e da decomposição tecidual vão agredir os tecidos periapicais que estão em íntimo contato com o canal radicular, via forame apical, dando início às periapicopatias ou alterações periapicais (Leonardi, Giovanini, Almeida, Schramm, \& Baratto-Filho, 2011).

Diante desta problemática, torna-se relevante avaliar o conhecimento dos cirurgiões-dentistas da atenção básica do Município de Arcoverde, Pernambuco, para o diagnóstico das doenças pulpares e periapicais.

\section{Metodologia}

Foi realizada uma pesquisa aplicada, quantitativa, descritiva e transversal (Pereira, Shitsuka, Parreira, \& Shitsuka, 2018), nas 22 Unidades Básicas de Saúde da Família, do município de Arcoverde, Pernambuco. A população foi constituída pelo total de cirurgiões-dentistas de ambos os sexos, idades variadas, diferentes aspectos socioeconômico-culturais, que atuam regularmente na atenção básica do município de Arcoverde/PE, e a amostra foi constituída de acordo com o cálculo amostral baseado na técnica para população finita, utilizando os critérios de inclusão.

Os critérios de inclusão abrangeram profissionais devidamente formados e registrados no Conselho Regional de Odontologia (CRO-PE) que atuavam na Atenção Básica do município de Arcoverde e que após informados sobre os objetivos e metodologia da pesquisa, concordaram em participar e assinaram o termo de consentimento livre e esclarecido. A presente pesquisa foi, inicialmente, enviada ao Comitê de Ética em Pesquisa da Universidade de Pernambuco (CEP) com o protocolo de aprovação de número 2.108.050, seguindo as diretrizes e normas estabelecidas pela Resolução 466/12 do Conselho Nacional de Saúde.

Foi feito um teste piloto em um grupo menor de dentistas e depois o instrumento foi enviado para o estatístico, para ser realizado tanto a validação como a confiabilidade. Depois de realizada a análise da validação e confiabilidade, o questionário foi aplicado nos demais profissionais. Além disso, muitos itens do questionário já tinham sido utilizados e validados no Programa Nacional de Melhoria do Acesso e de Qualidade dos Centros de Especialidades Odontológicas (PMAQ-CEO). Os cirurgiões-dentistas responderam o questionário estruturado combinando perguntas abertas, que admitem diferentes respostas dos pesquisados, e fechadas do tipo múltipla escolha, nas quais assinalavam apenas uma alternativa (resposta simples) ou mais de uma alternativa (respostas múltiplas), em escala de Likert. As questões abordavam as atitudes, condutas e critérios para o diagnóstico das doenças pulpares e periapicais em sua prática clínica odontológica e foram divididas nos seguintes temas: perfil socioprofissional dos participantes da pesquisa, frequência de realização do diagnóstico das doenças pulpares e periapicais, autoavaliação dos conhecimento dos aspectos clínicos e de diagnóstico das condições endodônticas, 
capacidade de interpretação dos dados semiológicos (aspectos clínicos e radiográficos das doenças pulpares e periapicais e respostas dos testes semiotécnicos) e disponibilidade dos recursos semiotécnicos no serviço.

Os dados obtidos foram organizados e categorizados para a obtenção das frequências das respostas do questionário, armazenados em programa específico para gerenciamento (Microsoft Excel® 2010. Redmond, WA, EUA) e submetidos à análise estatística descritiva, além de serem analisados por meio de tabelas de distribuição de frequência e apresentados em porcentagens.

\section{Resultados e Discussão}

Diante de uma urgência odontológica, o cirurgião-dentista precisa estar capacitado para executar o procedimento mais adequado para cada caso, estando atento aos sinais clínicos e sintomas das patologias orofaciais mais comuns encontradas nesses atendimentos (Macêdo, Oliveira, Gomes, dos Santos Barbosa, \& Medeiros, 2015). Visto que as urgências odontológicas constituem uma realidade no cotidiano do atendimento odontológico, é necessário que o cirurgião-dentista esteja capacitado para identificar as principais patologias pulpares e periapicais que ocorrem no serviço de Atenção Primária à Saúde (APS) (De Oliveira, Montagner, \& Fontanive, 2019).

As características socioprofissionais dos 18 cirurgiões-dentistas que atuam nas UBS e que participaram deste estudo são apresentadas na Tabela 1.

Tabela 1: Perfil socioprofissional dos participantes da pesquisa.

\begin{tabular}{|c|c|c|c|}
\hline $\begin{array}{l}\text { Tipo de vínculo } \\
\text { Empregatício }\end{array}$ & $\begin{array}{c}\text { Cirurgiões-dentistas das } \\
\text { UBSF }\end{array}$ & Possui pós-graduação & $\begin{array}{c}\text { Cirurgiões-dentistas das } \\
\text { UBSF }\end{array}$ \\
\hline & $\%$ & & $\%$ \\
\hline Concurso & $77.8 \%$ & Sim & $88.9 \%$ \\
\hline Contrato & $22.2 \%$ & Não & $11.1 \%$ \\
\hline \multirow[t]{2}{*}{ Grau de escolaridade } & $\begin{array}{c}\text { Cirurgiões-dentistas das } \\
\text { UBSF }\end{array}$ & $\begin{array}{c}\text { tempo de trabalho no } \\
\text { UBSF }\end{array}$ & $\begin{array}{c}\text { Cirurgiões-dentistas das } \\
\text { UBSF }\end{array}$ \\
\hline & $\%$ & & $\%$ \\
\hline Especialização & $50 \%$ & Menos de 3 anos & $66,66 \%$ \\
\hline Aperfeiçoamento & $44,4 \%$ & 3 a 10 anos & $22,22 \%$ \\
\hline Capacitação & $16,6 \%$ & Mais de 10 anos & $11,11 \%$ \\
\hline Mestrado & - & & \\
\hline Doutorado & - & & \\
\hline
\end{tabular}

Fonte: Autores.

Dentre os 18 profissionais participantes do estudo, 88,9\% possui pós-graduação, o que demonstra ser um grupo bem qualificado, admitidos por concurso público $77,8 \%$, fator que facilita o engajamento nas estratégias do programa de saúde da 
família e por ter vínculo estável, há um aumento do interesse na melhoria do serviço. O tempo de atuação mais frequente destes profissionais nas UBSFs, foi menos de 3 anos $66,66 \%$, o que proporciona experiência e aptidão para análise das práxis.

A figura a seguir, apresenta a frequência de diagnóstico das condições endodônticas evidenciadas em dentes permanentes nos últimos 12 meses, durante o atendimento nas UBSFs.

Figura 1: Estimativa das condições endodônticas verificadas em dentes permanentes nos últimos 12 meses, durante os atendimentos nas UBSF.

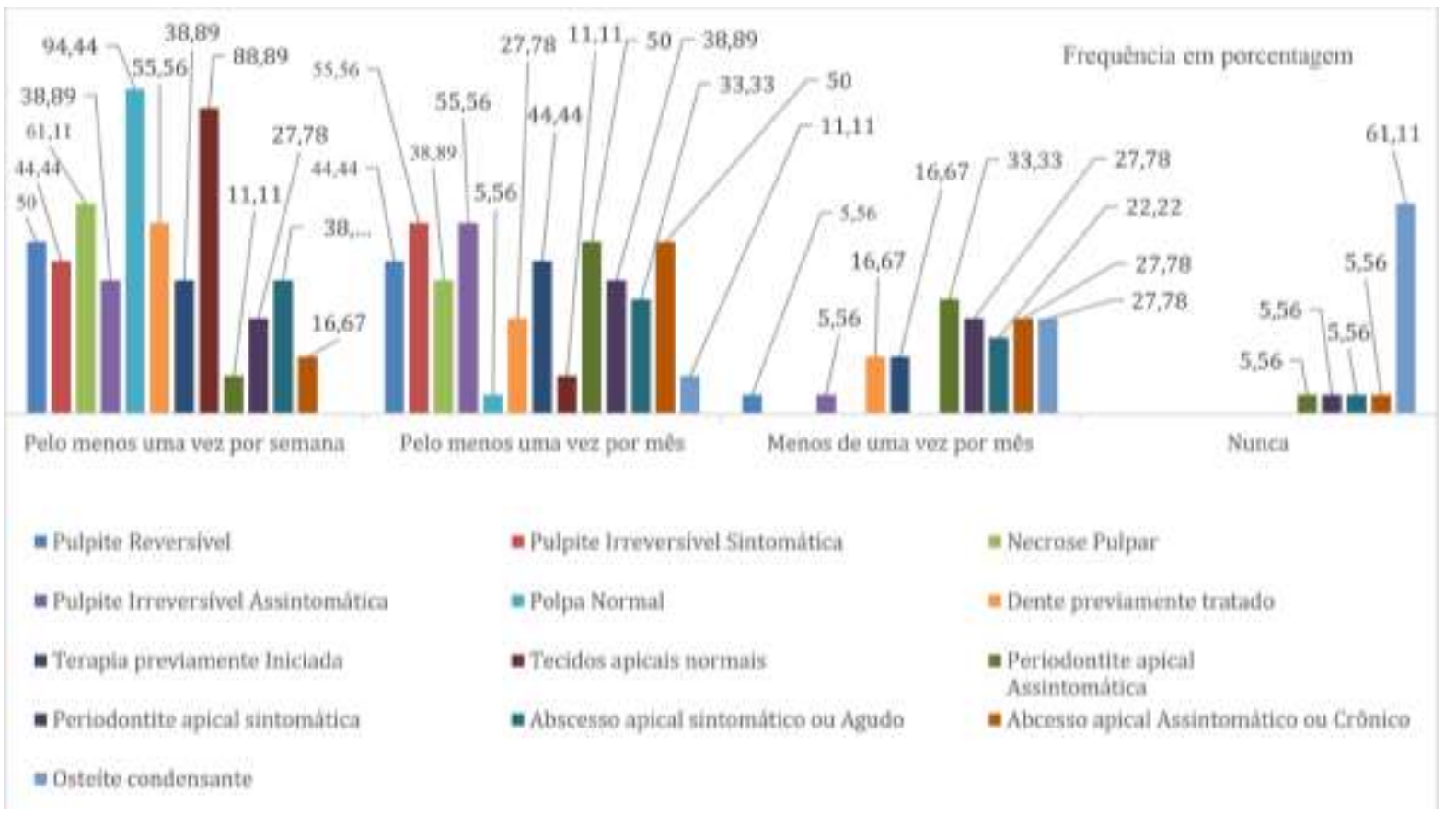

Fonte: Autores.

Dados de estudos prévios revelam que problemas odontológicos constituem o terceiro motivo de procura por serviços de saúde (Pessoa, 2016), sendo as alterações pulpares e periapicais parte desse universo, o que justifica a elevada frequência do diagnóstico destas patologias também no município de Arcoverde, confirmados pelos participantes da pesquisa. No entanto, estima-se que este número seja maior, pois, os dados coletados apontam lacunas de conhecimento na identificação e interpretação dos dados clínicos e radiográficos para determinação do correto diagnóstico.

A queixa principal é parte integrante no diagnóstico do doente e deve ser sempre registrada pelas próprias palavras, passando depois para as questões relativas da doença atual, como por exemplo: "Há quanto tempo está com essa dor?"; "O dente dói quando come algo frio ou quente?", sendo essencial para o correto diagnóstico a correlação entre sintomatologia dolorosa espontânea e contínua (Ribeiro, 2015; Lopes \& Siqueira, 2015; Barbin, Spanó, Matos, \& Schnorrenberger, 2016). No entanto, apenas dados subjetivos informados pelo paciente durante a anamnese, não são suficientes. (Ribeiro, 2015; Lopes \& Siqueira, 2015). Necessitando a associação da anamnese (da história do caso do paciente), da semiotécnica (dos recursos para coleta dos sinais e sintomas), da semiologia (da análise dos sinais e sintomas) e da propedêutica (da interpretação dos sinais e sintomas), fundamentais para o planejamento e intervenção clínica, determinantes para a resolutividade da morbidade do paciente, além de proporcionar, nos casos de urgência, o alívio da dor, bem como, condições para recuperação emocional, muitas vezes presente nos casos de pulpopatias e periapicopatias agudas (Barbin et al., 2016).

A Tabela 2 apresenta os resultados da autoavaliação dos profissionais em relação a sua aptidão para o reconhecimento dos aspectos clínicos e de diagnóstico das condições pulpares e periapicais. 
Tabela 2: Estimativa da autoavaliação dos profissionais referentes aos aspectos clínicos das seguintes condições pulpares e periapicais.

\begin{tabular}{|c|c|c|c|c|c|}
\hline Diagnóstico & $\begin{array}{c}\text { Discorda } \\
\text { fortemente } \\
(\%)\end{array}$ & $\begin{array}{l}\text { Discorda } \\
(\%)\end{array}$ & $\begin{array}{c}\text { Concorda } \\
(\%)\end{array}$ & $\begin{array}{c}\text { Concorda } \\
\text { fortemente } \\
(\%)\end{array}$ & $\begin{array}{c}\text { Não tem } \\
\text { certeza } \\
(\%)\end{array}$ \\
\hline Polpa normal & - & - & 72.22 & 27.78 & - \\
\hline Pulpite reversível & - & - & 66.67 & 22.22 & 11.11 \\
\hline $\begin{array}{l}\text { Pulpite irreversível } \\
\text { sintomática }\end{array}$ & - & 11.11 & 66.67 & 22.22 & - \\
\hline $\begin{array}{l}\text { Pulpite irreversível } \\
\text { assintomática }\end{array}$ & - & 11.11 & 61.11 & 16.67 & 11.11 \\
\hline Necrose pulpar & - & 5.56 & 61.11 & 33.33 & - \\
\hline $\begin{array}{c}\text { Terapia previamente } \\
\text { iniciada }\end{array}$ & - & 11.11 & 66.67 & 16.67 & 5.56 \\
\hline $\begin{array}{l}\text { Dente previamente } \\
\text { tratado }\end{array}$ & - & 5.56 & 66.67 & 22.22 & 5.56 \\
\hline $\begin{array}{c}\text { Tecidos apicais } \\
\text { normais }\end{array}$ & - & - & 72.22 & 27.78 & 5.56 \\
\hline $\begin{array}{l}\text { Periodontite apical } \\
\text { sintomática }\end{array}$ & - & 16.67 & 61.11 & 16.67 & 5.56 \\
\hline $\begin{array}{l}\text { Periodontite apical } \\
\text { assintomática }\end{array}$ & - & 16.67 & 55.56 & 16.67 & 11.11 \\
\hline $\begin{array}{c}\text { Abscesso apical } \\
\text { sintomático ou agudo }\end{array}$ & - & 5.56 & 72.22 & 22.22 & - \\
\hline $\begin{array}{l}\text { Abscesso apical } \\
\text { assintomático ou } \\
\text { crônico }\end{array}$ & - & 5.56 & 72.22 & 22.22 & - \\
\hline Osteíte condensante & - & 27.78 & 50.00 & - & 22.22 \\
\hline
\end{tabular}

Fonte: Autores.

As Tabelas 3, 4 e 5 apresentam as respostas dos profissionais relacionadas a interpretação dos dados semiológicos conhecimento dos aspectos clínicos das doenças pulpares e periapicais, conhecimento dos aspectos radiográficos das doenças pulpares e periapicais e conhecimento das respostas aos testes semiotécnicos. 
Tabela 3: Respostas dos profissionais em relação à sintomatologia das condições pulpares e periapicais.

\begin{tabular}{|c|c|c|c|c|c|c|c|}
\hline Diagnóstico & $\begin{array}{c}\text { Dor } \\
\text { aguda } \\
(\%)\end{array}$ & $\begin{array}{c}\text { Dor } \\
\text { localizada } \\
(\%)\end{array}$ & $\begin{array}{c}\text { Dor } \\
\text { moderada } \\
(\%)\end{array}$ & $\begin{array}{c}\text { Dor } \\
\text { difusa } \\
(\%)\end{array}$ & $\begin{array}{c}\text { Dor } \\
\text { contínua } \\
(\%)\end{array}$ & $\begin{array}{c}\text { Dor } \\
\text { pulsátil } \\
(\%)\end{array}$ & $\begin{array}{c}\text { Ausente } \\
(\%)\end{array}$ \\
\hline Polpa normal & - & - & 72.22 & 27.78 & - & - & 100.00 \\
\hline $\begin{array}{l}\text { Pulpite } \\
\text { reversível }\end{array}$ & 38.89 & 66.67 & 38.89 & 5.56 & 5.56 & 16.67 & 5.56 \\
\hline $\begin{array}{c}\text { Pulpite } \\
\text { irreversível } \\
\text { sintomática }\end{array}$ & 77.78 & 44.44 & - & 38.89 & 61.11 & 61.11 & - \\
\hline $\begin{array}{c}\text { Pulpite } \\
\text { irreversível } \\
\text { assintomática }\end{array}$ & - & 5.56 & 5.56 & - & - & 5.56 & 88.89 \\
\hline Necrose pulpar & 11.11 & 22.22 & 11.11 & 5.56 & 5.56 & 5.56 & 77.78 \\
\hline $\begin{array}{l}\text { Terapia } \\
\text { previamente } \\
\text { iniciada }\end{array}$ & - & 33.33 & 16.67 & - & 5.56 & - & 72.22 \\
\hline $\begin{array}{l}\text { Dente } \\
\text { previamente } \\
\text { tratado }\end{array}$ & 5.56 & 11.11 & 5.56 & - & 11.11 & 5.56 & 72.22 \\
\hline $\begin{array}{c}\text { Tecidos apicais } \\
\text { normais }\end{array}$ & - & 5.56 & - & - & - & 5.56 & 88.89 \\
\hline $\begin{array}{l}\text { Periodontite } \\
\text { apical } \\
\text { sintomática }\end{array}$ & 33.33 & 50.00 & 27.78 & 22.22 & 5.56 & 16.67 & 5.56 \\
\hline $\begin{array}{l}\text { Periodontite } \\
\text { apical } \\
\text { assintomática }\end{array}$ & 5.56 & 11.11 & 5.56 & - & - & 5.56 & 88.89 \\
\hline $\begin{array}{l}\text { Abscesso apical } \\
\text { sintomático ou } \\
\text { agudo }\end{array}$ & 50.00 & 38.89 & 5.56 & 38.89 & 27.78 & 38.89 & 5.56 \\
\hline $\begin{array}{l}\text { Abscesso apical } \\
\text { assintomático } \\
\text { ou crônico }\end{array}$ & - & 5.56 & 11.11 & 5.56 & - & 5.56 & 77.78 \\
\hline $\begin{array}{c}\text { Osteíte } \\
\text { condensante }\end{array}$ & 5.56 & 22.22 & 11.11 & 5.56 & 5.56 & - & 55.56 \\
\hline
\end{tabular}

Fonte: Autores.

Em relação aos dados clínicos, e especificamente relacionados à sintomatologia dolorosa das doenças pulpares e periapicais e suas condições de duração, intensidade, frequência e localização, a média de acertos dos participantes deste estudo foi de 36,56\%, considerado um percentual insuficiente. No âmbito clínico, a exploração da sintomatologia dolorosa, como forma de avaliação da vitalidade pulpar, deve constituir rotina no exame dentário. Por ser a polpa dental um tecido bastante vascularizado, a dor é o principal sintoma frente às alterações pulpares. Por isto, diversos autores defendem uma classificação clínica com base na sintomatologia dolorosa para tais alterações (Medeiros et al, 2017; Silva et al., 2008).

$\mathrm{O}$ desconhecimento pelos profissionais também ficou evidente para as alterações crônicas, que por sua natureza inflamatória, não se caracteriza clinicamente por dor ou sintomatologia espontânea, apenas provocada em alguns casos, e nas periapicopatias sintomáticas, nas quais não houve convicção dos participantes da presença da dor espontânea, característica marcante das alterações inflamatórias agudas de origem periapical, por ser um local de limitada expansão e drenagem do exsudato inflamatório (Lopes \& Siqueira, 2015). 
Tabela 4: Respostas dos profissionais em relação aos aspectos radiográficos das condições pulpares e periapicais.

\begin{tabular}{|c|c|c|c|c|c|c|c|}
\hline Diagnóstico & $\begin{array}{c}\text { Normal } \\
(\%)\end{array}$ & $\begin{array}{c}\text { Espessamento } \\
\text { do espaço } \\
\text { periodontal } \\
(\%)\end{array}$ & $\begin{array}{c}\text { Comunicação } \\
\text { câmara pulpar/ } \\
\text { cavidade oral } \\
(\%)\end{array}$ & $\begin{array}{c}\text { Área } \\
\text { radiolúcida } \\
\text { com aumento } \\
\text { irregular do } \\
\text { canal } \\
\text { radicular }(\%)\end{array}$ & $\begin{array}{l}\text { Rarefação } \\
\text { óssea (\%) }\end{array}$ & $\begin{array}{c}\text { Perda de } \\
\text { lâmina dura, } \\
\text { espessamento } \\
\text { apical ou zona } \\
\text { radiolucente } \\
(\%)\end{array}$ & $\begin{array}{c}\text { Obliteração } \\
\text { do espaço } \\
\text { pulpar }(\%)\end{array}$ \\
\hline Polpa normal & 100 & - & - & - & - & - & - \\
\hline $\begin{array}{l}\text { Pulpite } \\
\text { reversível }\end{array}$ & 83.33 & 16.67 & 22.22 & - & - & 5.56 & - \\
\hline $\begin{array}{l}\text { Pulpite } \\
\text { irreversível } \\
\text { sintomática }\end{array}$ & 38.89 & 61.11 & 44.44 & 5.56 & - & - & - \\
\hline $\begin{array}{c}\text { Pulpite } \\
\text { irreversível } \\
\text { assintomática }\end{array}$ & 27.78 & 61.11 & 38.89 & 11.11 & 5.56 & 16.67 & - \\
\hline $\begin{array}{l}\text { Necrose } \\
\text { pulpar }\end{array}$ & - & 88.89 & 61.11 & 16.67 & 38.89 & 27.78 & - \\
\hline $\begin{array}{c}\text { Terapia } \\
\text { previamente } \\
\text { iniciada }\end{array}$ & 22.22 & 16.67 & 44.44 & 33.33 & 11.11 & 5.56 & \\
\hline $\begin{array}{l}\text { Dente } \\
\text { previamente } \\
\text { tratado }\end{array}$ & 22.22 & - & 22.22 & 5.56 & - & - & 61.11 \\
\hline $\begin{array}{l}\text { Tecidos } \\
\text { apicais } \\
\text { normais }\end{array}$ & 94.44 & - & - & - & - & 5.56 & - \\
\hline $\begin{array}{l}\text { Periodontite } \\
\text { apical } \\
\text { sintomática }\end{array}$ & 5.56 & 77.78 & 5.56 & 11.11 & 27.78 & 55.56 & 5.56 \\
\hline $\begin{array}{c}\text { Periodontite } \\
\text { apical } \\
\text { assintomática }\end{array}$ & 16.67 & 38.89 & 5.56 & 11.11 & 33.33 & 50.00 & - \\
\hline $\begin{array}{c}\text { Abscesso } \\
\text { apical } \\
\text { sintomático ou } \\
\text { agudo }\end{array}$ & 11.11 & 44.44 & 27.78 & 27.78 & 22.22 & 72.22 & - \\
\hline $\begin{array}{c}\text { Abscesso } \\
\text { apical } \\
\text { assintomático } \\
\text { ou crônico }\end{array}$ & 5.56 & 38.89 & 27.78 & 27.78 & 50.00 & 72.22 & - \\
\hline $\begin{array}{c}\text { Osteíte } \\
\text { condensante }\end{array}$ & 11.11 & 33.33 & - & - & 44.44 & 27.78 & 11.11 \\
\hline
\end{tabular}

Fonte: Autores.

Em relação às características radiográficas, apenas 35,07\% dos aspectos imaginológicos considerados corretos foram assinalados pelos clínicos da atenção básica do município, considerando mais um resultado deficiente do conhecimento dos critérios essenciais para o diagnóstico endodôntico. O maior destaque foi para os casos de necrose pulpar, periodontite apical assintomática, abscesso agudo e crônico. Isso é um fator preocupante, pois o artifício da imagem radiográfica é um notável recurso complementar de exame para se obter o diagnóstico, orientar o tratamento e fornecer dados essenciais. 
Frequentemente, constitui o único meio para detecção e observação de estruturas anormais que ainda não foram apreciadas no exame clínico (Barbin et al., 2012).

Tabela 5: Respostas dos profissionais em relação à resposta aos testes semiotécnicos para cada condição endodôntica.

\begin{tabular}{|c|c|c|c|c|c|c|c|}
\hline Diagnóstico & $\begin{array}{c}\text { Positivo } \\
\text { ao frio } \\
(\%)\end{array}$ & $\begin{array}{c}\text { Positivo ao } \\
\text { calor }(\%)\end{array}$ & $\begin{array}{c}\text { Positivo a } \\
\text { palpação }(\%)\end{array}$ & $\begin{array}{c}\text { Positivo a } \\
\text { trepidação } \\
(\%)\end{array}$ & $\begin{array}{c}\text { Positivo a } \\
\text { percussão } \\
\text { vertical } \\
(\%)\end{array}$ & $\begin{array}{c}\text { Positivo a } \\
\text { percussão } \\
\text { horizontal }(\%)\end{array}$ & $\begin{array}{c}\text { Ausente } \\
(\%)\end{array}$ \\
\hline Polpa normal & 50.00 & 33.33 & 5.56 & 5.56 & 11.11 & 11.11 & 55.56 \\
\hline $\begin{array}{l}\text { Pulpite } \\
\text { reversível }\end{array}$ & 88.89 & 33.33 & 5.56 & 11.11 & 5.56 & 5.56 & 16.67 \\
\hline $\begin{array}{l}\text { Pulpite } \\
\text { irreversível } \\
\text { sintomática }\end{array}$ & 66.67 & 88.89 & 27.78 & 16.67 & 44.44 & 27.78 & - \\
\hline $\begin{array}{c}\text { Pulpite } \\
\text { irreversível } \\
\text { assintomática }\end{array}$ & 22.22 & 27.78 & - & - & 27.78 & 16.67 & 50.00 \\
\hline $\begin{array}{l}\text { Necrose } \\
\text { pulpar }\end{array}$ & - & - & 16.67 & 5.56 & 33.33 & 16.67 & 72.22 \\
\hline $\begin{array}{c}\text { Terapia } \\
\text { previamente } \\
\text { iniciada }\end{array}$ & - & - & - & - & 16.67 & 5.56 & 88.89 \\
\hline $\begin{array}{l}\text { Dente } \\
\text { previamente } \\
\text { tratado }\end{array}$ & 5.56 & - & - & 5.56 & 5.56 & - & 88.89 \\
\hline $\begin{array}{l}\text { Tecidos } \\
\text { apicais } \\
\text { normais }\end{array}$ & 22.22 & 5.56 & - & 5.56 & 5.56 & 5.56 & 77.78 \\
\hline $\begin{array}{l}\text { Periodontite } \\
\text { apical } \\
\text { sintomática }\end{array}$ & - & 5.56 & 27.78 & 22.22 & 61.11 & 66.67 & 16.67 \\
\hline $\begin{array}{l}\text { Periodontite } \\
\text { apical } \\
\text { assintomática }\end{array}$ & 5.56 & 5.56 & - & 5.56 & 16.67 & 27.78 & 72.22 \\
\hline $\begin{array}{c}\text { Abscesso } \\
\text { apical } \\
\text { sintomático ou } \\
\text { agudo }\end{array}$ & 11.11 & 22.22 & 55.56 & 22.22 & 88.89 & 66.67 & 11.11 \\
\hline $\begin{array}{c}\text { Abscesso } \\
\text { apical } \\
\text { assintomático } \\
\text { ou crônico }\end{array}$ & - & - & 27.78 & 11.11 & 22.22 & 16.67 & 72.22 \\
\hline $\begin{array}{c}\text { Osteíte } \\
\text { condensante }\end{array}$ & - & 5.56 & 5.56 & - & 16.67 & 5.56 & 72.22 \\
\hline
\end{tabular}


Os testes semiotécnicos auxiliam de forma contundente no diagnóstico e constituem a forma mais confiável para a obtenção da real condição do elemento dentário, todavia, na avaliação das interpretações destes testes, apenas 35,69\% foi a média de respostas corretas assinaladas pelos cirurgiões-dentistas participantes, o que repercute negativamente na possibilidade de elaboração das hipóteses diagnósticas (Santos; Veloso; Temóteo \& Brito, 2011; Silva et al., 2008).

Apesar de vários autores pactuarem da mesma opinião, que o uso de testes elétricos, térmicos e de palpação ajudam a estabelecer um diagnóstico subjetivo, porém nenhum destes é totalmente seguro, por causa da inexistência de sinal ou sintoma que designe a morbidade da polpa com exatidão (Silva et al, 2008). Diante das pulpites reversíveis, não houve unanimidade nas respostas corretas aos testes térmicos de sensibilidade ao frio, que deve ser utilizado para simular a dor provocada (Medeiros et al., 2010; Lopes \& Siqueira, 2015; Galara, \& Dorn, 2011). Em relação à necrose pulpar, que não apresenta nenhuma resposta aos testes, sendo considerado um diagnóstico fácil, apesar do alto percentual de profissionais que responderam corretamente, ainda houve respostas incorretas, correlacionando esta alteração a sintomatologia à palpação, trepidação e percussão vertical (Lopes \& Siqueira, 2015).

A pulpite irreversível assintomática não apresenta nenhuma resposta a testes com exceção do teste de percussão vertical, e metade dos profissionais responderam corretamente. Talvez, ainda ocorram dúvidas em relação às respostas dos testes neste tipo de alteração por ser uma condição não tão comum, mais presente em indivíduos jovens com atividade de cárie intensa (Lopes \& Siqueira, 2015).

Quanto à polpa normal que está em perfeita vitalidade respondendo aos testes térmicos e de trepidação, surpreendentemente 10 pesquisados assinalaram dor "ausente" correspondendo a 55,56\% dos participantes. Nesta condição, a vitalidade da polpa permite a resposta dolorosa a estes testes, porém dependendo da intensidade e do declínio da sensação dolorosa, podemos inferir que a polpa está sem alterações (Lopes \& Siqueira, 2015; Galara \& Dorn, 2011).

Nas situações de tecido periapical normal, mesmo com 14 profissionais assinalando "ausente", correspondendo a $77,78 \%$, ainda tivemos respostas positivas aos testes térmicos como se o tecido pulpar estivesse presente. Sabe-se que para uma alteração periapical surgir, já houve a completa degeneração da polpa com a passagem de microrganismos pelos condutos principais (Torres, 2017). O mesmo ocorreu para os casos de abscesso agudo.

A média dos acertos (Quadro 1), conforme gabarito pré-estabelecido das respostas dos participantes relacionadas a capacidade de interpretação dos dados semiológicos (aspectos clínicos e radiográficos das doenças pulpares e periapicais e respostas dos testes semiotécnicos) foram categorizadas utilizando os seguintes scores: insuficiente, regular ou suficiente.

Quadro 1: Distribuição dos Scores e categorização.

\begin{tabular}{|cccc|}
\hline Categorização & Score & $\begin{array}{c}\text { \% de profissionais } \\
\text { que assinalaram }\end{array}$ & Participantes \\
\hline Insuficiente & 0 a 5 & 0 a $50 \%$ & 18 \\
Regular & Acima de 5 a 7 & Acima de 50 a $70 \%$ & - \\
Suficiente & Acima de 7 a 10 & Acima de 70 a $100 \%$ & - \\
\hline
\end{tabular}

Fonte: Autores.

Apesar da maioria dos profissionais se sentirem aptos para o diagnóstico das alterações pulpares e periapicais, de maneira geral, a pesquisa revelou que o conhecimento dos critérios necessários para o seu diagnóstico pelos cirurgiõesdentistas da atenção básica do município de Arcoverde, é insuficiente. Esse resultado reforça os achados encontrados na literatura de que o conhecimento dos cirurgiões-dentistas acerca de diagnósticos e condutas em situações de urgências endodônticas é limitado. Dessa forma, é necessária a atualização permanente, para que o cirurgião-dentista atenda os casos de 
Research, Society and Development, v. 10, n. 4, e43510414055, 2021

(CC BY 4.0) | ISSN 2525-3409 | DOI: http://dx.doi.org/10.33448/rsd-v10i4.14055

urgência endodônticas de modo mais resolutivo, sendo esta necessidade identificada desde a graduação (Augusto, 2016; Souza, 2016)

A Figura 2 apresenta o percentual de acertos de cada questão pelo total dos participantes.

Figura 2: Distribuição da média percentual de acertos das questões referentes ao conhecimento dos aspectos clínicos, radiográficos e da interpretação das respostas dos testes semiotécnicos.

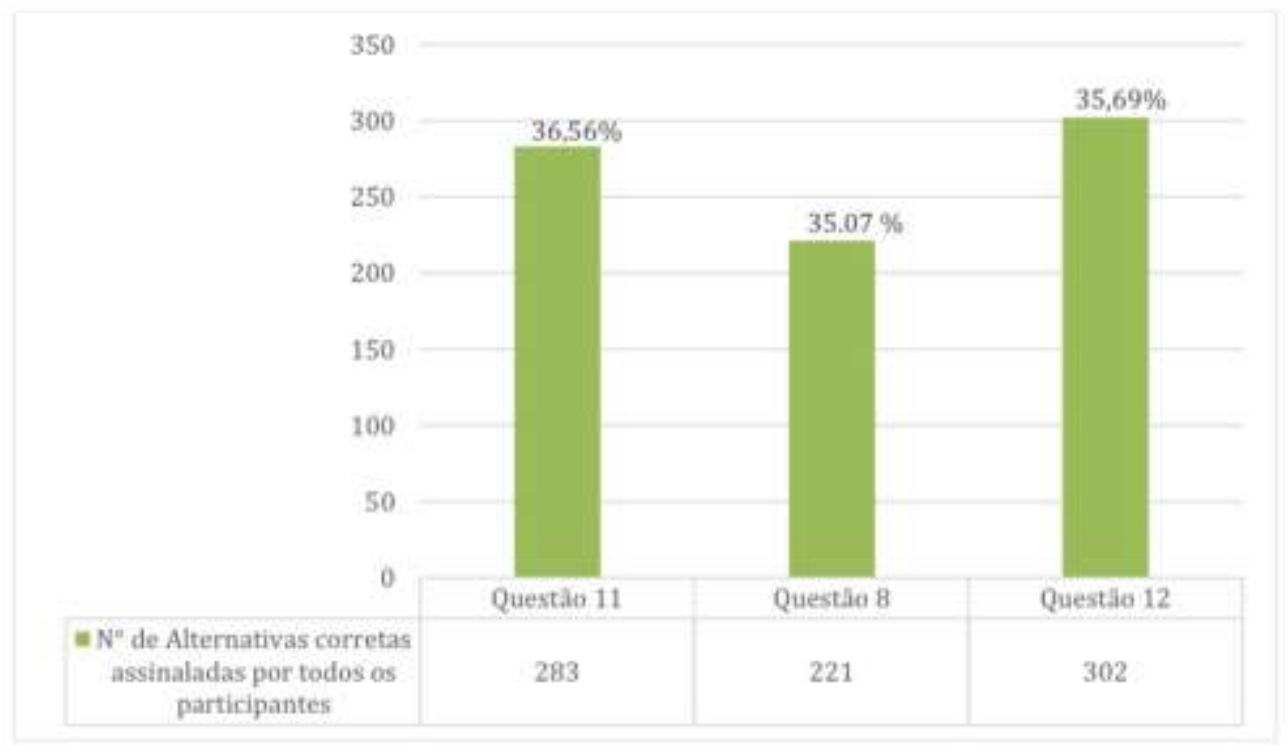

Fonte: Autores.

Nas Tabelas 6 e 7 são apresentadas a frequência de utilização de recursos semiotécnicos para o diagnóstico das condições endodônticas e a periodicidade de solicitação dos exames radiográficos pelos participantes da pesquisa, respectivamente. 
Tabela 6: Estimativa da frequência de utilização de recursos para o diagnóstico da condição pulpar e periapical pelos profissionais.

\begin{tabular}{|c|c|c|c|c|}
\hline & $\begin{array}{l}\text { Pelo menos } \\
\text { uma vez por } \\
\text { semana }(\%)\end{array}$ & $\begin{array}{l}\text { Pelo menos uma } \\
\text { vez por mês }(\%)\end{array}$ & $\begin{array}{c}\text { Menos de uma } \\
\text { vez por mês }(\%)\end{array}$ & $\begin{array}{c}\text { Nunca } \\
(\%)\end{array}$ \\
\hline $\begin{array}{c}\text { Inspeção clínica } \\
\text { visual }\end{array}$ & 100.00 & - & - & - \\
\hline Exame radiográfico & 72.22 & 27.78 & - & - \\
\hline Teste térmico ao frio & 33.33 & 11.11 & 11.11 & 44.44 \\
\hline $\begin{array}{l}\text { Teste térmico com } \\
\text { uso de } \\
\text { diclorodifluormetano }\end{array}$ & - & - & 11.11 & 88.89 \\
\hline $\begin{array}{c}\text { Teste térmico ao } \\
\text { calor }\end{array}$ & 5.56 & - & 16.67 & 77.78 \\
\hline Teste elétrico & - & - & - & 100.00 \\
\hline Teste de cavidade & 66.67 & 16.67 & 11.11 & 5.56 \\
\hline $\begin{array}{c}\text { Teste de percussão } \\
\text { vertical }\end{array}$ & 100.00 & - & - & - \\
\hline $\begin{array}{l}\text { Teste de percussão } \\
\text { horizontal }\end{array}$ & 100.00 & - & - & 5.56 \\
\hline $\begin{array}{l}\text { Sondagem } \\
\text { periodontal }\end{array}$ & 27.78 & 33.33 & 11.11 & 27.78 \\
\hline Teste anestésico & 33.33 & 11.11 & 11.11 & 44.44 \\
\hline Transiluminação & 5.56 & - & 5.56 & 88.89 \\
\hline
\end{tabular}

Fonte: Autores.

A radiografia também é de grande importância, para a análise da morfologia do sistema de canais radiculares e em certos casos para a avaliação da viabilidade, ou não, do tratamento endodôntico nesse mesmo dente. Contudo, diante das limitações e subjetividade das imagens fornecidas por este exame, devem ser apenas utilizadas como meios complementares importantes para a investigação do diagnóstico, pois quando utilizadas de forma isolada colaboram a interpretações precoces e diagnósticos incorretos (Ribeiro, 2015). 
Tabela 7: Periodicidade de solicitação das seguintes técnicas radiográficas.

\begin{tabular}{|c|c|c|c|c|}
\hline & $\begin{array}{c}\text { Pelo menos uma vez por } \\
\text { semana } \\
(1)\end{array}$ & $\begin{array}{c}\text { Pelo menos uma vez por } \\
\text { mês } \\
(2)\end{array}$ & $\begin{array}{c}\text { Menos de uma vez por } \\
\text { mês } \\
(3)\end{array}$ & $\begin{array}{c}\text { Nunca } \\
\text { (4) }\end{array}$ \\
\hline & $\%$ & $\%$ & $\%$ & $\%$ \\
\hline Periapical & 83,33 & 16,67 & - & - \\
\hline Interproximal & 11,11 & 11,11 & 27,78 & 50,00 \\
\hline Panorâmica & 16,67 & 22,22 & 55,56 & 5,56 \\
\hline Tomografia & - & - & 16,67 & 83,33 \\
\hline
\end{tabular}

Fonte: Autores.

Dentre as variadas técnicas radiográficas, a radiografia periapical é a de primeira escolha em casos de alterações pulpares e periapicais, podendo também ser empregadas as tomadas oclusal e panorâmica, sendo essa última alternativa indicada em casos de pacientes com limitação de abertura bucal. (Rodrigues, Cangussu, \& Figueiredo, 2015). Na presente pesquisa, 83,33\% dos profissionais solicitam pelo menos uma vez por semana a radiografia periapical, ou seja, nem todos requisitam este recurso importantíssimo de auxílio no diagnóstico. Talvez, por não terem o equipamento de raio x à disposição nas unidades de atendimento. Porém, em estudos preliminares de levantamento epidemiológico, há uma alta prevalência de casos com presença de algum tipo de lesão periapical, cujos diagnóstico diferencial com dentes portadores de necrose pulpar, apenas pode ser esclarecido com auxílio da radiografia, sendo um indício de falhas na determinação do diagnóstico (Pessoa, 2016).

A deficiente disponibilidade de recursos nas UBSFs, evidenciadas nos dados deste estudo, como a ausência do gás diclorodifluormetano que inviabiliza a realização dos testes térmicos, contribuem para as inconsistências de interpretação das respostas aos testes semiotécnicos, associado a baixa realização ou solicitação do exame radiográfico periapical, por parte dos profissionais. Pois, as alterações pulpares e periapicais possuem difícil diagnóstico por não conseguirmos visualizar de forma direta clinicamente o tecido alterado (Lopes \& Siqueira, 2015; Barbin at al., 2012; Silva et al., 2008).

Em decorrência disso, o paciente é prejudicado, por não obter um diagnóstico preciso, e influencia na tomada de decisão do profissional para a escolha do tratamento conservador ou radical endodôntico, o que repercute na vida útil do elemento dentário e negativamente no fluxo assistencial do serviço, pois muitos casos poderiam ser solucionados na própria unidade e são encaminhados para o nível secundário, prorrogando uma solução que poderia ser imediata, deixando o paciente vulnerável para o agudecimento dos processos crônicos ou evolução da doença, além de aumentar o custo para o serviço (Barbin et al., 2012; Santos et al., 2011; de Medeiros, Pinto, Rosa, Habitante, \& Almeida, 2010).

O estudo indica que há necessidade premente da qualificação e instrumentalização dos profissionais que compõem as equipes de saúde da família do município de Arcoverde/PE, além do incremento dos recursos para favorecer a construção do diagnóstico endodôntico, baseado nas características clínicas e radiográficas dos processos inflamatórios pulpares e periapicais e na indicação, execução e correta interpretação das respostas aos testes semiotécnicos, contribuindo para uma atenção à saúde bucal de melhor qualidade para a população.

Fica evidente que o cirurgião-dentista deve estar preparado para lidar com urgências endodônticas desde sua formação acadêmica. Para isso, atualizações constantes sobre o tema são necessárias, contribuindo para a ampliação dos seus conhecimentos e a resolutividade da assistência odontológica. 


\section{Conclusão}

De acordo com a metodologia empregada, pode-se concluir que a maioria dos cirurgiões-dentistas da atenção básica do município de Arcoverde/PE possuem conhecimento insuficiente e utilizam de forma deficiente os critérios de diagnóstico das doenças pulpares e periapicais.

\section{Referências}

Augusto, S. M. (2016) Conhecimento e práticas de cirurgiões-dentistas da estratégia saúde da família acerca das urgências endodônticas [Monografia de Graduação em Odontologia]. Campina Grande: Universidade Estadual da Paraíba.

Albuquerque, L. A. D., Archer, C. E., Souza, R. M. S. D., Travassos, R. M. C., Gomes, S. G. F., \& Santos, R. A. D. (2011). Prevalência de doenças pulpares e periapicais na clínica de especialização em endodontia da FOP/UPE. Revista de Cirurgia e Traumatologia Buco-maxilo-facial, 11 (1), 77-83.

Barbin, E. L., Spanó, J. C., Matos, M, \& Schnorrenberger, R. (2012). Metodologia e Sistemática de Diagnóstico das Pulpopatias e Periapicopatias. Plataforma de Ensino Continuado de Odontologia e Saúde (PECOS).

Campos, C. N., Campos, A. D. S. O., \& Bellei, M. D. C. (2018). Tecnologia a serviço da Endodontia: avanços no diagnóstico e tratamento de canais radiculares. $H U$ rev, 55-61.

Caputo, I. G. C., Bazzo, G. J., Silva, R. H. A. D., \& Daruge Júnior, E. (2010). Vidas em risco: emergências médicas em consultório odontológico. Revista de Cirurgia e Traumatologia Buco-maxilo-facial, 10(3), 051-058.

Góes Silva, L. D., Albergaria, S., Gonçalves, P. S., \& dos Santos, J. N. (2008). Diagnóstico endodôntico: comparação entre aspectos clínicos e histológicos. $R G O, 56(1), 59-65$.

Marques, A. C. R. (2014). Endodontia: sessão única versus múltiplas sessões

Medeiros, J. M. F., Pinto, C. A., da Rosa, L. C. L., Habitante, S. M., de Almeida, E. T. D. C., \& Zollner, N. A. (2017). Avaliação da escolha dos testes de sensibilidade pulpar por clínicos gerais da cidade de Taubaté. Revista de Odontologia da Universidade Cidade de São Paulo, 22(1), 30 -38.

Oliveira, M. M., Montagner, F., \& Fontanive, V. N. (2019). Conhecimento de cirurgiões-dentistas de Atenção Primária à Saúde acerca de urgências endodônticas. Revista da Faculdade de Odontologia-UPF, 24(2), 192-197.

Garala, M., \& Dorn, S. O. (2011). Contemporary endodontic evaluation and diagnosis: implications for evidence-based endodontic care. Today's FDA: official monthly journal of the Florida Dental Association, 23(3), 43-5.

Leonardi, D. P., Giovanini, A. F., Almeida, S., Schramm, C. A., \& Baratto-Filho, F. (2011). Alterações pulpares e periapicais. RSBO (Online), 8(4), 47-61.

Lopes, H., \& Siqueira, J. (2015). Endodontia-Biología e Técnica. Ed.

Macêdo, P. A. D. S. A., de Oliveira, M. E. M., Gomes, T. N., dos Santos Barbosa, J., \& Medeiros, E. F. (2015). Principais Patologias Orofaciais Recorrentes em Serviços de Urgências Odontológicas: Uma Revisão da Literatura. ID on line Revista De Psicologia, 9(27), $167-177$.

Pereira, A. S., Shitsuka, D. M., Parreira, F. J., \& Shitsuka, R. (2018). Metodologia da pesquisa científica. UFSM. https://repositorio. ufsm. br/bitstream/handle/1/15824/Lic_Computacao_Metodologia-Pesquisa-Cientifica.pdf.

Pessoa, A. L. L. (2016). Estudo da prevalência das doenças pulpo periapicais e do perfil dos pacientes atendidos no curso de especialização da UFRN (Bachelor's thesis, Universidade Federal do Rio Grande do Norte).

Ribeiro, J. F. M. (2015). Urgências endodônticas: Protocolo de atuação

Rodrigues, J. E. M., Cangussu, I. S., \& de Figueiredo, N. F. (2015). Abscesso periapical versus periodontal: Diagnóstico dife rencial-Revisão de literatura. Arquivo Brasileiro de Odontologia, 11(1), 5-9.

Santos, K. S. A. D., Veloso, O. L. L., Temóteo, L. M., \& Brito, L. N. S. (2011). Concordância diagnóstica em Endodontia em clínicas odontológicas. RGO. Revista Gaúcha de Odontologia (Online), 59(3), 365-371.

Souza, D. P. (2016) Conhecimentos dos estudantes de odontologia sobre urgências endodônticas [Monografia de Graduação em Odon tologia]. Campina Grande: Universidade Estadual da Paraíba.

Torres, M. P. (2017). Pulpite aguda: etiologia, diagnóstico e tratamento 